\title{
Trophic transfer of pesticides: the fine line between predator-prey regulation and pesticide-pest regulation
}

\section{Virgile Baudrot ${ }^{*, 1,2}$, Javier Fernandez-de-Simon ${ }^{1}$, Michael Coeurdassier ${ }^{1}$, Geoffroy Couval $^{3}$, Patrick Giraudoux ${ }^{1}$, Xavier Lambin ${ }^{4}$}

${ }^{1}$ Chrono-environment, UMR UFC/CNRS 6249 aff. INRA, Université de Bourgogne Franche-Comté, 25030 Besançon, France

2 BioSP, INRAE, 84000 Avignon, France

3 FREDON Bourgogne Franche-Comté, Espace Valentin Est, 12, Rue de Franche-Comté - Bât E, 25480 Ecole-Valentin, France

${ }^{4}$ School of Biological Sciences, University of Aberdeen, Zoology Building, Tillydrone Avenue, Aberdeen AB24 2TZ, Scotland, UK

* Corresponding authors: virgile.baudrot@posteo.net

Running Title Sensitive line of predator/pesticide pest-regulation

\begin{abstract}
1. Understanding pesticide impacts on populations of target/non-target species and communities is a challenge to applied ecology. When predators that otherwise regulate pest densities ingest prey contaminated with pesticides, this can suppress predator populations by secondary poisoning. It is, however, unknown how species relationships and protocols of treatments (e.g. anticoagulant rodenticide (AR)) interact to affect pest regulation.

2. To tackle this issue, we modelled a heuristic non-spatialized system including montane water voles, specialist vole predators (stoats, weasels), and a generalist predator (red fox) which consume voles, mustelids and other prey. By carrying out a broad-range sensitivity analysis on poorly known toxicological parameters, we explored the impact of 5 farmer functional responses (defined by both AR quantity and threshold vole density above which AR spreading is prohibited) on predator-prey interactions, AR transfer across the trophic chain and population effects.

3. Spreading AR to maintain low vole densities suppressed mustelid and fox populations, leading to vole population dynamics being entirely regulated by AR use. Such vole-suppression treatment regimes inhibited predation ecosystem services and promoted pesticide dependence.
\end{abstract}


4. Keeping vole density below acceptable bounds by spreading AR while maintaining sufficient voles as prey resources led to less AR being applied and extended periods without AR in the environment, benefiting predators while avoiding episodes with high vole density. This may meet farm production interests while minimizing the impact on mustelid and fox populations and associated ecosystem processes. These alternating phases of mustelids and farmer regulation highlight the consequence of intraguild relationship where mustelids may rescue foxes from poisoning. Both global and wide-range sensitivity analysis illustrate the tightrope between predator-prey regulation and pesticide-pest regulation.

5. Synthesis and applications Different pesticide protocols lead to a rich variety of predatorprey dynamics in agro-ecosystems. Our model reveals the need to maintain refuges with sufficient non-poisoned voles for sustaining specialist mustelids, to conserve the predator community given the potential of secondary poisoning with rodenticides. We suggest that long periods without pesticide treatment are essential to maintain predator populations, and that practices of pesticides use that attempt to permanently suppress a pest over a large scale are counterproductive.

Keywords biodiversity conservation; secondary poisoning; cyclic fluctuations; pesticides; cascade effects; ecosystem service; sensitivity analysis ; ecological control

\section{Introduction}

Since the "green revolution" following the 1950s, pesticides use has increased to control pests damaging properties, public health or crops (Tilman et al., 2002). Pesticides usage is varyingly triggered by the perception/estimation of pest densities. Natural enemies (e.g., predators, parasites, competitors) also reduce pest densities and hence may preclude the need for using pesticides (Michalko and Pekár, 2017). Natural enemies are, however, also affected by pesticides, either by direct exposure, through ingestion of contaminated prey (Berny, 2007) or indirectly through cascading consequences of effects of resource depletion (Halstead et al., 2014). Thus, under some regimes of pesticides use, pest populations only become regulated by pesticides once predators have collapsed. To preserve ecosystem health and the services that predators provide through regulation of pest densities, we need to assess the feasibility and benefit of pesticide treatment regimes in their ability to control pest species with minimal damage to predators (Halstead et al., 2014). It is however empirically challenging to assess the overall impact of pesticide treatments on the dynamics of species linked by trophic interactions. In this context, process-based models describing simplified scenarios are powerful tools to reveal hidden patterns by disentangling processes emerging from pesticide impacts on predator-prey systems (e.g., Baudrot et al. (2018)).

Voles and other grassland rodent species undergo multi-annual population cycles (e.g., Krebs (2013)). At their peaks, they may attain extremely high densities, causing substantial damage to grass/cultivated crops and forestry and conflicts with humans (Delattre and Giraudoux, 2009). Farmers worldwide expand financial resources to purchase and spread anticoagulant rodenticides 
(hereafter AR), hoping to reduce vole populations and damages, and increase profits despite the investment required (Stenseth et al., 2003). They do so according to protocols, equivalent to farmer functional responses (hereafter FFR), that involve varying amounts of AR spread in response to different thresholds in vole densities.

Voles and many small rodents are perceived as pests, but they are also keystone species, crucial to the functioning of grassland and forest ecosystems, as well as being the prey of numerous predators, including species of conservation concern (Delibes-Mateos et al., 2011; Coeurdassier et al. 2014). Their population cycles create pulses of resources crucial to the viability of a wide range of resident predators and the aggregation of mobile avian vole predators (Korpimaki and Norrdahl, 1991). The smallest mustelids (e.g. weasels Mustela nivalis) are specialist vole predators. Their numerical response has been shown theoretically to be necessary for generating predator-prey cycles (Hanski et al., 1991). They are said to be responsible for driving 3-to-5-year vole cycles in Fennoscandia (the specialist predation hypothesis) (Hanski et al., 1991). Generalist resident predators like foxes (Vulpes vulpes) are expected to have regulatory and limiting effects on voles, owing to dietary plasticity that slows down vole population increase at low density (Hanski et al., 1991). Foxes do not show numerical responses to vole abundance (Weber et al., 2002) but they influence the food chain through occasional killing and consumption of bite-sized mustelids. Mustelids form a small proportion of fox diet (0-10\%) but their offtake could represent a significant portion of the population (reviewed in Lambin (2018)).

Anticoagulant rodenticides are non-selective toxicants with deleterious effects on non-target fauna (e.g. Coeurdassier et al. (2014)). Despite AR being exclusively licensed for rodent control, a large number of predator species are secondarily exposed to AR (Sánchez-Barbudo et al., 2012). Consumption of dead and sub-lethally intoxicated voles reduced fox abundance in farmland in eastern France (Jacquot et al., 2013) and ARs caused short-term declines in stoats in New Zealand (Alterio, 1996). Rodent-eating mustelid populations are affected by ARs given the pervasive levels of contamination reported (McDonald et al., 1998). Thus, there is little doubt ARs use inadvertently depresses predator populations. As predators likely limit vole populations, it is essential to understand when AR use becomes counterproductive by altering the pest population dynamics, producing more frequent outbreaks and high residual vole abundance.

With the aim of understanding the potentially complex interactions between prey that are perceived as pest, predators and farmers spreading rodenticide in response to vole abundance and their functional responses, we studied a simplified system inspired by cyclically fluctuating montane water voles (Arvicola scherman), small mustelids (stoats, weasels) that mostly eat voles (specialists), and foxes (generalists), with voles and mustelids as food items. We used a process-based model using differential equations to explore 5 FFR types of AR spread, combining population dynamics, predator-prey interactions and rodenticide transfer across the trophic chain. Model parameters and FFR were inspired by farming systems in the Jura Mountains, Franche-Comté (France), the region of Comté cheese production. In Franche-Comté, farmers shifted from polyculture to almost exclu- 
sively grass production for milk used to produce cheese from the early 1970s (Giraudoux et al., 1997). Due to recurrent vole outbreaks and damages to grasslands, massive rodenticide treatments were implemented from the early 80s with consequences on non-target wildlife. Practices were developed technically under pressure from public opinion, farmer unions and farmer technical organizations collaborating with researchers to find treatment regimes with less harmful consequences for biodiversity (Delattre and Giraudoux, 2009).

Hence, our main objective was to explore the properties of FFR in relation to varying population sensitivity to AR on the global tri-trophic population dynamics made of vole outbreak frequencies and guild of interacting predators.

\section{Materials and Methods}

We specified and parameterized the tri-trophic system of voles-mustelids-foxes with use of AR by farmers in response to vole density. We considered several FFR depending on the AR amount and vole threshold triggering AR spreading to assess AR upward transfer through the trophic web. In all cases, parameterization units are on hectare ${ }^{-1}$ and day $^{-1}$.

\subsection{Model for the tri-trophic dynamic}

We considered a tri-trophic system described by Figure 1 and equations (1-5); parameterization is provided in Table 1 . Voles, denoted $V$, were the primary prey, Mustelids, $M$, intermediate predators, and Foxes, $F$, were top predators, consuming voles and mustelids. For each species, the instantaneous variation of population size over time is:

$$
\left\{\begin{aligned}
\frac{d V}{d t} & =V r_{V}\left(1-\frac{V}{K_{V}}\right)-\Phi_{V, M}(V) M-\Phi_{V, F}(V, M) F \\
\frac{d M}{d t} & =\varepsilon_{M} \Phi_{V, M}(V) M-m_{M} M-\Phi_{M, F}(V, M) F \\
\frac{d F}{d t} & =F r_{F}\left(1-\frac{F}{K_{F}}\right)
\end{aligned}\right.
$$

The vole population followed a logistic growth rate, with $r_{V}$ the maximal reproduction rate, fixed at $r_{V}=\ln (2 \times 600) / 365$ per day, since montane water vole populations can increase from 0 to 600 individuals ha ${ }^{-1}$ or more (Giraudoux et al., 1997), resulting in the equilibrium density being fixed at $K_{V}=600$ individuals. The vole population was preyed upon by mustelid and fox populations. The vole consumption rate at different vole densities was described by functional responses ( $\Phi_{V, M}$ for mustelids, $\Phi_{V, F}$ for foxes), see equation (2). We assumed small mustelids behave as specialist predators (King and Powell, 2006), we considered a numerical response linearly dependent on the functional response with parameter $\varepsilon_{M}$ (dimensionless) as conversion efficiency of prey into newborn predator (see Supporting Information - Appendix S1). The mustelid background mortality rate (i.e. all other reasons of death: ageing, disease, etc.) was $m_{M}$ parameterized has the inverse 
of life expectancy (Table 1). We assumed foxes had a logistic growth rate function, parameterized with maximal growth rate $r_{F}=\ln (3) / 365$ and equilibrium density $K_{F}=0.03$ individuals ha $^{-1}$ (Ruette et al., 2003) without numerical response (Weber et al., 2002).

Since we treated small mustelids as vole specialist predators, we assumed a Holling Type II functional response with attack rate $a_{M}$ and handling time $h_{M}$ (equation (2)). We then represented foxes feeding on voles and mustelids by a multi-species functional response derived from Holling Type III, referring to generalist feeding behaviour (Baudrot et al., 2016). For that, we denoted $a_{V F}$ and $a_{M F}$ the fox attack rate on voles and mustelids respectively. The parameter $h_{F}$ was the handling time for foxes.

$$
\begin{aligned}
& \Phi_{V, M}(V) M=\frac{a_{M} V}{1+h_{M} a_{M} V} \\
& \Phi_{V, F}=\frac{a_{V F} V}{a_{V F} V+a_{M F} F} \times \frac{\left(a_{V F} V+a_{M F} M\right)^{2}}{1+h_{F}\left(a_{V F} V+a_{M F} M\right)^{2}} \\
& \Phi_{M, F}(V, M) F=\frac{a_{M F} V}{a_{V F} V+a_{M F} F} \times \frac{\left(a_{V F} V+a_{M F} M\right)^{2}}{1+h_{F}\left(a_{V F} V+a_{M F} M\right)^{2}}
\end{aligned}
$$

Parameterization of functional responses was estimated to fit the daily satiation level of predators for handling times, and the observed 5-6 year vole cycles for attack rates (Table 1). We assumed foxes spent longer searching for voles than mustelids, based on each species diet and daily number of individuals captured. Therefore, $a_{V F}$ was considered larger than $a_{M F}$ and selected to produce 6-year vole cycles without AR.

\subsection{Model with rodenticide}

Figure 1 represents the whole study system. Rodenticide is spread in grasslands during treatments, denoted $T_{\text {Broma }}(V)$, triggered by vole density $V$. Firstly, baits $\left(50 \mathrm{mg} \mathrm{kg}^{-1}\right.$ of bromadiolone, hereafter AR) were spread in grasslands at quantity 7.5 to $20 \mathrm{~kg} \mathrm{ha}^{-1}$ day $^{-1}$. Notation day ${ }^{-1}$ stands because of the daily time resolution. Such quantity, $C$, was available for voles, and a proportion disappeared in the environment at rate $k_{0}$ (set at $k_{0}=0.0815$ ) (Sage et al., 2008). The proportion consumed per vole, with rate $\kappa(C)$, was assumed to be an increasing function. The function $\kappa(C)$ was characterized by a maximum ingestion $M_{i n}$, and a half-saturation constant for ingestion $D_{i n}$ in $\left[\mathrm{mg} \mathrm{kg}^{-1}\right]$ :

$$
\kappa(C)=\frac{M_{i n} \times C}{D_{i n}+C}
$$

For the toxicokinetics of AR (i.e. internal compound dynamics) leading to AR concentrations in animal body (voles, mustelids and foxes), we considered an uptake without biotransformation and time-regulated distribution, (i.e. AR concentration in the body of animals was instantly homogeneous) and that the whole body was consumed or scavenged without selection/rejection of tissues-organs. We also assumed disappearance including excretion of the parent compound and metabolisation, and that metabolites were non-toxic and/or excreted in the scats. For the toxi- 
cokinetics of AR ingested by voles, a fraction $C_{V}$ was assumed to remain active and available to predators ingesting voles. The absorption rate of ARs $(\eta)$ exceeds $50 \%$ in less than 24h (Jacquot et al., 2013). The excretion rate from voles, $k_{\text {out }}, V$ was 0.4 day $^{-1}$ (Sage et al., 2008). The mortality rate through ARs was $\mu\left(D_{V}\right)$. Death through poisoning created a dead vole population $\left(V_{d}\right)$ with AR concentration $C_{V}$. Dead voles could either be scavenged by mustelids/foxes or decompose at rate $d$. We assumed AR in dead voles disappeared from the system when voles decomposed (assumed in one week: $d=1 / 7$ day $^{-1}$ ). Mustelids could feed on live voles $V$, or non-decomposed dead voles $V_{d}$ and we assumed a Type II functional response adapted for a multi-species functional response (Baudrot et al., 2016). Mustelids ingested AR with absorption rate $\eta_{M}$ (ratio between biomasses of voles, $B_{V}$, and mustelids, $B_{M}$ ) and the total of ingested voles (alive $V$ and dead $V_{d}$ ) was defined by function $\Theta_{M}\left(V, V_{d}=\right.$ (Table 1$)$.

A fraction of AR ingested was accumulated in weasels while the rest was excreted with rate $k_{\text {out }, M}$. AR contaminating weasel though vole poisoning, is denoted $D_{M}$, induces lethal effect at rate $\mu_{M}\left(D_{M}\right)$, additive to natural mortality rate $m_{M}$. Parameter definitions are further detailed in Supporting Information (Appendix S1). AR was ingested by foxes with a rate proportional to the functional response of foxes to voles, dead voles and mustelids. Foxes also accumulated AR available in their prey, resulting in upward AR transfer in the trophic chain. Foxes accumulated AR in concentration $C_{F}$. A fraction of $\mathrm{AR}$ was excreted by foxes at rate $k_{\text {out }, F}$ at a rate between 0.38 and 0.72 day $^{-1}$ (Sage et al., 2010), and AR caused fox mortality at rate $\mu_{F}\left(D_{F}\right)$.

We used log-logistic equations for describing dose-dependent mortality of animals exposed to AR. Vole and predator mortality rates due to $\mathrm{AR} \mu_{X}\left(D_{X}\right)$ ( $X$ referring to the considered species) were expressed by equation (5):

$$
\mu_{X}\left(\zeta_{X}\right)=1-\frac{1}{1+\left(L D_{50} / \zeta_{X}\right)^{H}}
$$

The parameter $L D_{50}$ is the daily median lethal dose (50\% of population dying), parameter $H$ is the Hill's coefficient modulating the curve steepness (see Table 1). Parameterization of $L D_{50}$ is important since it defines the inflexion point of the ecotoxicological effect. While it is a classical parameter targeted in experiments, empirical estimates of its value are highly uncertain and indeed span different order of magnitude for the same species and under the same experimental design (Grolleau et al., 1989; Erickson and Urban, 2004; Sage et al., 2010; Karmaus et al., 2018). We therefore carefully explored this parameterization through a wide-range sensitivity analysis detailed here-after (see also Figure 2).

\subsection{The farmer functional responses (FFRs) explored through simulation}

We considered a range of realistic FFRs spanning treatments during vole outbreaks only and a precautionary approach in which treatments only takes place at intermediate or low vole density threshold. These scenarios are inspired by historic and contemporary protocols of bromadiolone 
use to control montane water voles in Franche-Comté, but also representative of practice globally (Delattre and Giraudoux, 2009): (A) a scenario without AR treatment, (B) a scenario with high vole density threshold triggering treatment (500 ind ha ${ }^{-1}$ ) and high AR amount per treatment (20 $\mathrm{kg} \mathrm{ha}^{-1}$ ), (C) a third scenario with intermediate triggering threshold (250 ind ha ${ }^{-1}$ ) and high AR per treatment (20 kg ha-1), (D) scenario with intermediate threshold (250 ind ha ${ }^{-1}$ ) and low AR amount (7.5 $\mathrm{kg} \mathrm{ha}^{-1}$ ) and (E) scenario with low threshold (50 ind ha ${ }^{-1}$ ) and low treatment (7.5 $\mathrm{kg} \mathrm{ha}^{-1}$ ). To check the influence of predators such as foxes and intraguild predation on the system dynamics, we also simulated scenarios with and without foxes. Our simulations tracked the linked vole-mustelid-fox dynamics for 40 years, after a "burn-in" period of 10 years to reduce dependency of results upon initial conditions, to observe several vole cycles and to characterise AR effects on these species population dynamics. This burn-in period also had AR treatment triggered at specific vole densities and with a given rodenticide quantity for each FFR. The burn-in phase was selected according to a set of simulations with different initial conditions. Those simulations showed that in a given FFR (i.e., same threshold of vole density and amount of AR spread), the dynamics of the population were converging toward a similar pattern.

\subsection{Numerical Simulation and Sensitivity Analysis}

All numerical analysis have been done using the open language $\mathrm{R}$ and particularly the ODE solver package deSolve (Soetaert et al., 2010). Model implementation and code to run analysis are available on a Github repository (Baudrot et al., 2020).

We applied a wide-range sensitivity analysis on lethal dose parameters (i.e. $L D_{50}$ ) for voles, mustelids and foxes, to explore changes in dynamical patterns. While wide-range variations of each parameter is likely to impact the global pattern, the lethal dose parameter is a direct characteristic of the toxicological part of the model. The variability of $L D_{50}$ from in vivo acute systemic toxicity studies under same experimental design spans 2 to 3 order of magnitude (see rat oral acute toxicity in Karmaus et al. (2018) ), which makes it challenging to assess risk with exposure models. Since the $L D_{50}$ parameter of fox appears to have low impact on dynamical pattern in our model, we evaluated only 4 different values (i.e. 0.5, 1.5, 2 and $7 \mathrm{ppm}$ ) which are consistent with a large range covering such a parameter (Erickson and Urban, 2004; Sage et al., 2010). For the prey and the specialist predator, this parameter was much more sensitive, so we explored 10 values for both, respectively in the range $[0.7-7] \mathrm{ppm}$ and $[1-25] \mathrm{ppm}$ following consistent ranges (Grolleau et al., 1989; Erickson and Urban, 2004). We classified the subsequent 1600 simulations according to the dynamical pattern of mustelids population: either "periodic regulation by mustelid" or "AR permanent regulation" where mustelids collapse.

Then, we performed a local sensitivity analysis to study changes in model outputs apportioned to small variation around model parameters input (Saltelli et al., 2019). We applied a first-order Sobol's sensitivity index $S_{i}$ defined as $S_{i}=\mathbb{V}\left(\mathbb{E}_{-i}\left(y \mid x_{i}\right)\right) / \mathbb{V}(y)$, where $\mathbb{V}(y)$ is the variance of $y$ when all factors are allowed to vary, $\left.\mathbb{E}_{x \sim i}\left(y \mid x_{i}\right)\right)$ the mean of $y$ when one factor is fixed (Sobol and Saltelli, 
1993). We defined the domain of variation with a beta distribution within an hyperspace of plus or minus $10 \%$ from the original value for all parameters. For output variable of local sensitivity analysis, we estimated the following cost functions: (i) Number of treatment events per FFR; (ii) Cumulative amount of AR (kg); (iii) Proportion of time when the AR-induced mortality of mustelids higher than 50\% (i.e. lethal exposure profile killing 50\% of mustelid population); (iv) Proportion of time when the mortality of mustelids was higher than $50 \%$ due to natural mortality (see Supporting Information - DATA available); (v) Proportion of time when the vole density was below 50 voles $\mathrm{ha}^{-1}$, as a proxy for time when forage grass grows with low herbivore influence; (vi) Mean vole, mustelid and fox densities.

\section{Results}

Allowing for mortality by predators ingesting AR-poisoned voles changed the outcome of predatorprey dynamics involving vole, mustelid and fox populations. Secondary poisoning led to a rich spectrum of emergent dynamics according to the FFR to vole abundance. Without AR (scenario A), vole dynamics were regulated by mustelid predation that gave rise to a 6-year cycles (Figure 3) typical of prey-specialist dynamics, while the generalist fox population remained at its carrying capacity (i.e., 0.03 ind ha ${ }^{-1}$, see Table 1). Based on this null model, we explored 4 scenarios of treatment described earlier and denoted respectively B, C, D and E, defined by the vole density threshold triggering treatment (respectively: 500, 250, 250 and 50 ind ha ${ }^{-1}$ ) and the AR amount per treatment (respectively: 20, 20, 7.5 and $7.5 \mathrm{~kg} \mathrm{ha}^{-1}$ ).

\subsection{Sensitivity analysis of model parameters}

The exploration of ecotoxicological uncertainties on population dynamics was performed for the lethal dose parameters $L D_{50}$ for voles, mustelids and foxes (see Figure 2). From the 1600 patterns explored two typical patterns emerged. On the one hand (i) vole dynamics were sequentially regulated by either AR treatments, which we refer to as farmer-regulated phase, or by mustelids, mustelids-regulated phase (see in Figure 3 panel c. B-2). This succession of farmer-regulated and mustelids-regulated phases are denoted "Mustelid periodic regulation" in Figure 2 (black pixels). On the other hand, (ii) vole dynamics were permanently regulated by AR treatments, because of crashes of the mustelid population we refer to as "Permanent AR regulation" in Figure 2 (grey pixels). We see that $L D_{50}$ for foxes does not change the general pattern of dynamics. Both scenario B and D exhibited the highest fractions of "Mustelid periodic regulation" compared to scenarios $\mathrm{C}$ and E. This illustrates the tight line that exists between resource and AR impact to benefit from predator regulation periods.

For other parameters, we performed a local sensitivity analysis where we computed a first order sensitivity index (Sobol and Saltelli, 1993; Saltelli et al., 2019) given the contribution of each parameter to the variance (in Figure 5 for scenario D - intermediate amount of $7.5 \mathrm{~kg} \mathrm{AR}$ at threshold 
density of 250 voles). Parameter values were moved within $+/-10 \%$ interval around the fixed value as defined in scenario D (see Table 1). Crossing all cost functions to test the sensitivity of model parameters (Figure 5), the influence of each parameter was quite homogeneous. There was no redundant or conversely any parameters with a dominant influence on model behaviour. The homogeneity of the sensitivity to parameters was particularly true for densities of predators to which we focused our attention.

\subsection{Population dynamics}

While both "Mustelid periodic regulation" and "Permanent AR regulation" pattern occurred under the 4 scenarios with AR (B, C, D and E) (see Figure 2), we illustrate in Figure 3 the most contrasting population dynamics for each of scenario in order to better describe their underlying mechanism. The boundary between contrasting dynamics lies for vole $L D_{50}$ around 1.5 to $2 \mathrm{ppm}$ and in the range 2 to 2.25 ppm for mustelid $L D_{50}$.

From Figure 3, we see that scenario B (high vole density threshold triggering treatment and high AR amount per treatment) and scenario D (intermediate threshold and low AR amount) accounts for the most of dynamics with sequential farmer-regulated phase and mustelids-regulated phase. Farmer-regulated periods started when densities of living voles triggered treatments. This produced sudden declines of live voles followed by increases of dead voles. However, the vole population recovered quickly which triggered repeated further treatments and pulses of availability of contaminated (both live and dead) voles. Also, in scenario D, vole declines were not as deep as when pulses of AR amount were high like in scenario B (see Supporting Information), owing to the reduced AR amount per treatment. Mustelids and sometimes foxes also experienced AR-induced declines during this period (Figure 3, c,f,g,h and Figure 4). For the predators, mustelid-regulated periods started when mustelid numbers grew slowly to a peak, which depressed vole density, precluding AR treatments and releasing the fox population from secondary poisoning, such that its abundance rebounded. Vole depletion by mustelids and subsequent mustelid declines allowed the vole population to grow again up to threshold densities that initiated a new period of regulation by farmers.

For scenario C (intermediate threshold and high AR), vole dynamics were mostly regulated by AR treatment only (e.g. Figure 3-e). Populations of live and dead voles experienced high frequency fluctuations driven by AR. As AR treatments were frequent, being triggered by vole peaks, contaminated dead voles were always abundant (see Supporting Information: peaks at 90 ind ha ${ }^{-1}$ ). With scenario E (low threshold, low AR) vole populations were maintained by farmers at around 50 voles ha $^{-1}$ (See Figure 3 and Supporting Information). The population of live voles was regulated by treatments. For most of simulations (except when vole $L D_{50}$ with high, Figure 2), whenever voles reached densities triggering treatment, predator populations experienced strong declines. However, fox densities were higher compared to scenario C (intermediate threshold, high AR) (see Supporting Information), reflecting the reduced amount of AR used $(7.5 \mathrm{~kg})$ and transferred to foxes as 
there were lower vole densities. The maximum numbers of dead voles under this scenario $\mathrm{E}$ was relatively low (highest around 15 ind ha ${ }^{-1}$ ) but, due to frequent treatments, there was a steady replenishment of contaminated dead voles. This, in turn, induced mustelid and fox mortality and population declines (Supporting Information). Additionally, low availability of live voles triggered small mustelids mortality through starvation, down to abundances similar to those resulting from AR use (Supporting Information).

\subsection{Influence of intra-guild predation}

Figure 4 shows the system dynamics under a scenario D, where successive farmer-regulated and mustelids-regulated phases occurred. This simulation shows that the removal of foxes did not eliminate the successions of mustelids-regulated and farmer-regulated phases. However, the mustelid regulated period allowed short-term peaks of voles, suggesting the emergence of a classical onepredator - one-prey cycles interrupted by a farmer-regulated period. Without foxes, population dynamics of mustelids presented a more chaotic behaviour, while it presented regular cyclic pattern with fox occurrence. Therefore, this simple model suggests a stabilizing role of a generalist predator (foxes in this system) during the mustelid-regulated period. At the end of the mustelid-regulated period, foxes strongly contributed to vole mortality and, to a lesser degree, mustelid mortality. Consequently, the removal of foxes implied less predation on voles during the mustelid regulated period, and short-term vole releases from mustelid predation. The 2-year rolling mean of vole density (blue lines in Figure (4) illustrates the change of regime from farmer-regulated to mustelids-regulated alternating phases. Indeed, the amplitude of averaged vole densities (i.e., the amplitude of vole cycles for the 2-year rolling mean) was relatively stable at the beginning of farmer regulation periods and then suddenly decreased to become minimal before sharply increasing, announcing a regime change. These changes in density amplitude may be used as an early-warning signal of the regime transition.

\section{Discussion}

Considering that rodenticide kills not only voles but also their predators through secondary poisoning, our models show that AR profoundly changes the modeled outcome of predator-prey dynamics involving vole, mustelid and fox populations beyond what mere intuition could elucidate. Our study reveals how the dual influences of the amount of pesticide spread and the vole density threshold triggering AR spread drive (i) pesticide spreading frequency, (ii) predation ecosystem service, and subsequently (iii) the control of pest outbreaks. Two types of a rich spectrum of emergent dynamics, including farmer or mustelid regulation changing from classical predator prey dynamics arose because poisoned voles acted as global stressor on the food chain. 


\subsection{Modelling farmer regulation into a classical predator-prey system}

The threshold functional response of farmers deciding when to apply varying amounts of rodenticides according to prevailing vole density was crucial in selecting the emergent ecosystem dynamics, resulting in much variability in ecosystem and conservation and farming production interests. In the idealised ecosystem our models depict, farmers spreading rodenticide not only depleted vole prey exploited by specialist and generalist predators but also created pulses of lethally or sublethally poisoned voles that subsequently poison their predators. Arguably this set of ecological interactions has similarities with circumstances where a pathogen affecting prey species also infects predators, as in the case with the flea vectored plague (Yersinia pestis) infecting prairie dogs (Cynomys spp.) and black footed ferrets (Mustela nigripes) in central US (Matchett et al., 2010). However, to our knowledge, the behaviour of such tri-trophic model with multiple reciprocal interactions has not been explored. This is despite the obvious relevance to the management of the globally widespread circumstances where keystone small mammals are poisoned and may secondarily poison their predators (Delibes-Mateos et al., 2011).

Under the "reference" scenario without AR spreading (A), we assumed a predator-prey cycle which is a plausible pattern thoroughly explored theoretically (Hanski et al., 2001), though with debated empirical support (Lambin, 2018). There is no controversy on the role of small mustelids tracking vole dynamics, though it is not yet well understood whether there is sufficient lag between predators and prey for predation to drive steep declines (King and Powell, 2006). Parameters of the reference scenario for our predator-prey model were biologically realistic and tuned to generate population fluctuations similar to those observed in the studied cyclic system (Delattre and Giraudoux, 2009). The addition of pulses of rodenticide and their toxicokinetics in vole and predators are based on previous experiments with bromadiolone, a widely used AR, ensuring biologically realistic functional forms and their parameterization. Irrespective of the FFR considered, the frequency of vole cycles dramatically increased compared to the reference scenario, except during mustelid-regulated phases emerging under some FFR scenarios.

\subsection{How specialist predators may protect generalists from poisoning}

An interesting model behaviour with farmer- and mustelid-regulated phases alternating with low frequency was seen with scenarios B (high vole threshold, high AR) and D (intermediate vole threshold, intermediate AR), and to a lesser extend scenario C (intermediate vole threshold, high AR) and rarely with scenario E (lowest vole density threshold). Such flipping between alternative states in population dynamics has been previously described in predator-prey model where weasels rely on a primary prey and entrain the dynamics of secondary prey (Hanski and Henttonen, 1996) but not for the kind of indirect interaction we explore here. It further demonstrates that adding biologically realistic complexity to simple models may drastically change the emergent properties of trophic interactions. From these scenarios, we understand that the emergence of successive farmer- and mustelid-regulated phases is neither driven by vole density threshold alone nor by AR amount, but 
instead by a subtle combination of both. Also, under scenarios with low $L D_{50}$ for fox, the modelling description of these patterns uncovered the dual key roles of mustelids on fox dynamics, as intraguild competitors and as a vector for poisoning. This led to a surprising form of facilitation for foxes: mustelids protect foxes from collapses. The establishment of such a response can be described in 3 steps. Firstly, low mustelid densities inhibit their regulation of voles and contribute to farmer AR use. In line with empirical evidence, the latter directly impacts foxes by poisoning (Jacquot et al., 2013). Secondly, fox predation on mustelids is reduced, and with an intermediate AR amount, this allows mustelids to slowly recover. Vole outbreaks and subsequently farmer AR treatments are then gradually delayed, benefitting mustelids recovery. This is the point of transition from farmer to mustelids regulation regimes, starting the third step: mustelids increase faster, suppressing vole densities and precluding the need for AR treatments, and eventually indirectly allowing the fox population growth.

Our findings that complexities in trophic interaction, induced by the poisoning of predator by poisoned prey, may cause the system to flip between alternative states is novel and robust. However, given we only explored deterministic and spatially homogeneous versions of our model, any inference on the frequency of flipping between states should be cautious given the inherent stochastic nature of natural and farmland environments. If such dynamics occur within real farming systems, flipping between states is unlikely to emerge with regularity where many other factors impact population dynamics. While generalists are known to have stabilising effect (Hanski et al., 1991), the benefit of specialist predators imparted to generalist predator and resulting increase in the prevalence of intraguild predation would be difficult to detect in empirical studies. Nevertheless, other generalist predators such as the endangered red kite (Milvus milvus) which feed on voles opportunistically, occupy areas with bromadiolone treatments and are also affected by rodenticides (Coeurdassier et al., 2014) and may therefore also benefit from the presence of mustelids in the ecosystem.

\subsection{How region-wide vole suppression may inhibit ecosystem services}

In many situations, notably scenario $\mathrm{E}$ and $\mathrm{C}$ and when vole and mustelids are highly sensitive to $\mathrm{AR}$, the whole system was solely driven by farmer regulation, whereby the chronic use of $\mathrm{AR}$ completely suppressed the pest-regulation ecosystem service of predators. It has previously been shown empirically that repeated rodenticide treatments are highly detrimental to the populations of predators and reduce their densities (Jacquot et al., 2013). Secondary poisoning of predators is an established reality (Berny, 2007). Through modelling, we formalised the insight that some poison deployment protocols, including those presently used in the empirical system which motivated our study, are counterproductive if employed on a large scale, suppressing natural predator regulation of pest rodents. It has been long known that poisoning rodents with AR permeates the food chain at peak abundance, achieves little in terms of protecting crops and may have strong deleterious impact (Olea et al., 2009). In Franche-Comté, a change in treatment protocols, from controlling 
voles at high densities to low-intermediate densities, has reduced the mortality of non-target species (including foxes) (Jacquot et al., 2013). Nevertheless, deployment regimes of pesticides that can contaminate the food chain should also include periods of time which permit predator populations to rebound and avoid extirpation from the ecosystem. We have shown that, over time, farmers who strictly maintain voles at low density thresholds would likely suppress predation services provided by vole predators and, in so doing, instigate pesticide dependence. In addition, small mammals like voles certainly have ecosystem functions. Our results also suggest that the presence of small mustelids in ecosystems is beneficial for biodiversity conservation (see above) and agriculture interests. Given the importance of vole cycles and their trophic interactions, it is desirable to maintain vole population fluctuations of sufficient amplitude to maintain ecosystem processes.

\subsection{Managing rodents and ecosystems}

Presently, in Franche-Comté, farmers relying on bromadiolone alone can only treat pre-emptively when voles are at low densities (scenario E) whereas those also using alternative methods (i.e., mechanical, not pesticide-based) are allowed to spread AR in low quantity up to intermediate vole densities (scenario D). Spreading AR in low quantity seems superficially desirable, but our heuristic model, assuming an idealised homogenous landscape, shows this is associated with frequent treatments. Consequently, it would induce a near permanent availability of a small number of intoxicated voles which, combined with low availability of non-contaminated voles, would reduce predator populations. Therefore, the extreme situation of using a low vole density threshold (scenario E) at a large scale is undesirable because it depletes the prey resources of foxes and mustelids and their populations. Triggering treatment at intermediate vole density with a low amount of AR (scenario D) allows for temporal refuges, i.e. longer periods free of rodenticide necessary for predator densities to rebound while simultaneously avoiding episodes with high vole density, as required by farm production interests. Under a landscape management approach, such temporal refuges could arguably be substituted for by spatial refuges, with parts of the landscape free of pesticides and fuel the recolonisation of the farming landscape by predator populations.

Our key result and the basis for management prescriptions is that allowing for refuges where voles are not poisoned and allowed to persist at medium-high densities such that they can be exploited by mustelids is crucial for predator population recovery and preserving the ecosystem services mustelids deliver. Treatment regimes allowing temporal and/or spatial refuges seems compatible with both conservation and farming interests. A critical insight is to avoid potential side effects of chronic low-dose AR prescription (e.g., depletion of community services, stimulation of resistances), as is well known with antibiotics, by demanding regularly long-term period without treatment. However, combining chronic treatments and long periods free of AR may be difficult to achieve in real systems. Our model only considers temporal refuges, and the conceptualization of untreated areas as equivalent to triggering treatment at intermediate vole density cannot provide guidance on the size of these spatial refuges. Nevertheless, while management of voles is 
implemented at the scale of fields, mustelids and foxes roams over much larger areas (King and Powell, 2006), such that large refuges with medium-high vole densities voles would be required while maintaining low vole density at local scale.

\subsection{Conclusion and perspectives}

Our process-based model revealed pesticides that permeate the food chain upward can lead to diverse population dynamics with alternative states regulated by predators and farmers. It also shows that the practice currently promoted to use low-dose AR treatments at low vole density could have the undesirable side-effects of leading to chronic application of AR on a large scale, in the absence of refuges, and the depletion of the vole predator community. This emerging question would benefit from a landscape modelling approach to characterize spatial refuges. An other prerequisite for this work becoming applied and guiding management practice would be to further explore toxocological lethal and sublethal (e.g. growth, reproduction, behaviour) effects of pesticides on population dynamics. We have also uncovered a counterintuitive mechanism whereby, owing to intraguild predation, mustelids could rescue foxes from poisoning. This suggest that contemporary Environmental Risk Assessment of pesticides that mostly consider one-species - one-compound experiments fail to capture the impact of pesticides on trophic links. Assessing risk at the ecosystem level is empirically challenging such that process-based modelling can play a critical role.

\section{Authors' contributions}

XL conceived the initial idea; all authors developed the concept; VB and JF developed the models and led manuscript writing; VB implemented the model and ran simulations. GC contributed treatment protocols; MC, JF and VB explored model parameters; XL, PG and MC contributed critically to drafts; all authors gave final approval for publication.

\section{Acknowledgments}

JF benefited from a Marie Skłodowska-Curie fellowship (European Commission, project "VOLES", 660718). VB was employed with this project funds. We are very grateful to Deon Roos for reviewing drafts. We thank Alessandro Massolo, Thibault Moulin and Francis Raoul for helpful suggestions. This work benefited from long-term data collected at Zone atelier (ILTER) Arc jurassien (http://zaaj.univ-fcomte.fr) and its financial support.

\section{DATA availability statement}

All code and data used for this manuscript are available on Github https://zenodo.org/badge/ latestdoi/233555669 (Baudrot et al., 2020). 


\section{References}

Alterio, N., 1996. Secondary poisoning of stoats (Mustela erminea), feral ferrets (Mustela furo), and feral house cats (Felis catus) by the anticoagulant poison, brodifacoum. New Zealand Journal of Zoology 23, 331-338.

Baudrot, V., Fritsch, C., Perasso, A., Banerjee, M., Raoul, F., 2018. Effects of contaminants and trophic cascade regulation on food chain stability: Application to cadmium soil pollution on small mammals-raptor systems. Ecological Modelling 382, 33-42.

Baudrot, V., Perasso, A., Fritsch, C., Giraudoux, P., Raoul, F., 2016. The adaptation of generalist predators' diet in a multi-prey context: insights from new functional responses. Ecology 97, $1832-1841$.

Baudrot, V., Fernandez-de Simon, J., Coeurdassier, M., Couval, G., Giraudoux, P., Lambin, X., 2020. Supporting Information Code and Figures on Github Repository - "Trophic transfer of pesticides: the fine line between predator-prey regulation and pesticide-pest regulation". URL: https:// doi.org/10.5281/zenodo.3606425, doi:10.5281/zenodo.3606425.

Berny, P., 2007. Pesticides and the intoxication of wild animals. Journal of veterinary pharmacology and therapeutics 30, 93-100.

Coeurdassier, M., Riols, R., Decors, A., Mionnet, A., David, F., Quintaine, T., Truchetet, D., Scheifler, R., Giraudoux, P., 2014. Unintentional wildlife poisoning and proposals for sustainable management of rodents. Conservation biology 28, 315-321.

Delattre, P., Giraudoux, P., 2009. Le campagnol terrestre: prévention et contrôle des populations. Editions Quae.

Delibes-Mateos, M., Smith, A.T., Slobodchikoff, C.N., Swenson, J.E., 2011. The paradox of keystone species persecuted as pests: a call for the conservation of abundant small mammals in their native range. Biological Conservation 144, 1335-1346.

Erickson, W.A., Urban, D.J., 2004. Potential risks of nine rodenticides to birds and nontarget mammals: a comparative approach. US Environmental Protection Agency, Office of Prevention, Pesticides and Toxic Substances.

Giraudoux, P., Delattre, P., Habert, M., Quéré, J., Deblay, S., Defaut, R., Duhamel, R., Moissenet, M., Salvi, D., Truchetet, D., 1997. Population dynamics of fossorial water vole (Arvicola terrestris scherman): a land use and landscape perspective. Agriculture, Ecosystems \& Environment 66, 47-60.

Grolleau, G., Lorgue, G., Nahas, K., 1989. Toxicité secondaire, en laboratoire, d'un rodenticide anticoagulant (bromadiolone) pour des prédateurs de rongeurs champêtres: buse variable (Buteo buteo) et hermine (Mustela erminea). EPPO Bulletin 19, 633-648. 
Halstead, N.T., McMahon, T.A., Johnson, S.A., Raffel, T.R., Romansic, J.M., Crumrine, P.W., Rohr, J.R., 2014. Community ecology theory predicts the effects of agrochemical mixtures on aquatic biodiversity and ecosystem properties. Ecology letters 17, 932-941.

Hanski, I., Hansson, L., Henttonen, H., 1991. Specialist predators, generalist predators, and the microtine rodent cycle. The Journal of Animal Ecology , 353-367.

Hanski, I., Henttonen, H., 1996. Predation on competing rodent species: a simple explanation of complex patterns. Journal of Animal Ecology , 220-232.

Hanski, I., Henttonen, H., Korpimäki, E., Oksanen, L., Turchin, P., 2001. Small-rodent dynamics and predation. Ecology 82, 1505-1520.

Jacquot, M., Coeurdassier, M., Couval, G., Renaude, R., Pleydell, D., Truchetet, D., Raoul, F., Giraudoux, P., 2013. Using long-term monitoring of red fox populations to assess changes in rodent control practices. Journal of Applied Ecology 50, 1406-1414.

Karmaus, A., Fitzpatrick, J., Allen, D., Patlewicz, G., Kleinstreuer, N., W, C., 2018. Variability of LD50 values from rat oral acute toxicity studies: Implications for alternative model development. US-EPA Science Inventory .

King, C.M., Powell, R.A., 2006. The natural history of weasels and stoats: ecology, behavior, and management. Oxford University Press.

Korpimaki, E., Norrdahl, K., 1991. Numerical and functional responses of kestrels, short-eared owls, and long-eared owls to vole densities. Ecology 72, 814-826.

Krebs, C.J., 2013. Population fluctuations in rodents. University of Chicago Press.

Lambin, X., 2018. The population dynamics of bite-sized predators: Prey dependence, territoriality, and mobility, in: Biology and Conservation of Musteloids. Oxford University Press (OUP), pp. $129-148$.

Matchett, M.R., Biggins, D.E., Carlson, V., Powell, B., Rocke, T., 2010. Enzootic plague reduces black-footed ferret (Mustela nigripes) survival in montana. Vector-Borne and Zoonotic Diseases $10,27-35$

McDonald, R., Harris, S., Turnbull, G., Brown, P., Fletcher, M., 1998. Anticoagulant rodenticides in stoats (Mustela erminea) and weasels (Mustela nivalis) in england. Environmental Pollution 103, $17-23$

Michalko, R., Pekár, S., 2017. The behavioral type of a top predator drives the short-term dynamic of intraguild predation. The American Naturalist 189, 242-253. 
Olea, P.P., Sánchez-Barbudo, I.S., Viñuela, J., Barja, I., Mateo-Tomás, P., Pineiro, A., Mateo, R., Purroy, F.J., 2009. Lack of scientific evidence and precautionary principle in massive release of rodenticides threatens biodiversity: old lessons need new reflections. Environmental Conservation $36,1-4$.

Ruette, S., Stahl, P., Albaret, M., 2003. Applying distance-sampling methods to spotlight counts of red foxes. Journal of Applied Ecology 40, 32-43.

Sage, M., Cœurdassier, M., Defaut, R., Gimbert, F., Berny, P., Giraudoux, P., 2008. Kinetics of bromadiolone in rodent populations and implications for predators after field control of the water vole, Arvicola terrestris. Science of the total environment 407, 211-222.

Sage, M., Fourel, I., Cœurdassier, M., Barrat, J., Berny, P., Giraudoux, P., 2010. Determination of bromadiolone residues in fox faeces by LC/ESI-MS in relationship with toxicological data and clinical signs after repeated exposure. Environmental research 110, 664-674.

Saltelli, A., Aleksankina, K., Becker, W., Fennell, P., Ferretti, F., Holst, N., Li, S., Wu, Q., 2019. Why so many published sensitivity analyses are false: A systematic review of sensitivity analysis practices. Environmental Modelling \& Software .

Sánchez-Barbudo, I.S., Camarero, P.R., Mateo, R., 2012. Primary and secondary poisoning by anticoagulant rodenticides of non-target animals in spain. Science of the Total Environment 420, 280-288.

Sobol, I., Saltelli, A., 1993. Sensitivity analysis for non-linear mathematical models. Mathematical Modeling \& Computational Experiment (English Translation) 1, 407-414.

Soetaert, K., Petzoldt, T., Setzer, R.W., 2010. Solving differential equations in r: Package desolve. Journal of Statistical Software 33, 1-25. URL: http://www.jstatsoft.org/v33/i09, doi:10. $18637 /$ jss.v033.i09.

Stenseth, N.C., Leirs, H., Skonhoft, A., Davis, S.A., Pech, R.P., Andreassen, H.P., Singleton, G.R., Lima, M., Machang'u, R.S., Makundi, R.H., et al., 2003. Mice, rats, and people: the bio-economics of agricultural rodent pests. Frontiers in Ecology and the Environment 1, 367-375.

Tilman, D., Cassman, K.G., Matson, P.A., Naylor, R., Polasky, S., 2002. Agricultural sustainability and intensive production practices. Nature 418, 671.

Weber, J.M., Aubry, S., Ferrari, N., Fischer, C., Feller, N.L., Meia, J.S., Meyer, S., 2002. Population changes of different predators during a water vole cycle in a central european mountainous habitat. Ecography 25, 95-101. 
Table 1 - Populations dynamics and toxicological parameters used in the model simulations. Notation ppm stands for parts-per-million $\left[\mathrm{mg} \mathrm{kg}^{-1}\right]$ and n.d. denotes a dimensionless parameter. See description in Methods.

\begin{tabular}{|c|c|c|c|}
\hline Parameters & Definitions & Units & Value \\
\hline \multicolumn{4}{|c|}{ Population dynamics parameters } \\
\hline$r_{V}$ & Maximal growth rate of voles & day $^{-1}$ & $\ln (2 \times 600) / 365$ \\
\hline$K_{V}$ & Carrying capacity of voles & ind $\mathrm{ha}^{-1}$ & 600 \\
\hline$a_{M}$ & Attack rate of mustelids on voles & day $^{-1}$ & $1 / 30$ \\
\hline$h_{M}$ & Handling time of mustelids on voles & day & $1 / 3.5$ \\
\hline$a_{V F}$ & Attack rate of foxes on voles & day $^{-1}$ & $1 / 10$ \\
\hline$a_{M F}$ & Attack rate of foxes on mustelids & day $^{-1}$ & $1 / 10 \times 80 / 300$ \\
\hline$h_{F}$ & Handling time of foxes on voles & day & $1 / 6$ \\
\hline$\varepsilon_{M}$ & Conversion efficiency of ingested food & n.d. & 0.0025 \\
\hline$m_{M}$ & Natural mortality rate of mustelids & day $^{-1}$ & $1 /(0.8 \times 365)$ \\
\hline$r_{F}$ & Maximal growth rate of foxes & day $^{-1}$ & $\ln (3) / 365$ \\
\hline$K_{F}$ & Carrying capacity of foxes & ind ha $\mathrm{h}^{-1}$ & 0.03 \\
\hline$B_{V}$ & Mean biomass of a vole individual & $\mathrm{g}$ & 80 \\
\hline$B_{M}$ & Mean biomass of a mustelid individual & $\mathrm{g}$ & 300 \\
\hline$B_{F}$ & Mean biomass of a fox individual & $g$ & 5800 \\
\hline$d$ & Degradation rate of dead vole & ind.day $^{-1}$ & $1 / 7$ \\
\hline \multicolumn{4}{|c|}{ Toxicological parameters } \\
\hline$T_{\text {broma }}(V)$ & Farmer input AR function of vole density & $\mathrm{mg} \mathrm{ha}{ }^{-1}$ day $^{-1}$ & Scenarios \\
\hline$k_{0}$ & Disappearance of AR in the field & day $^{-1}$ & $(0.106+0.057) / 2$ \\
\hline$M_{i n}$ & Maximal intake rate of vole & ppm day ${ }^{-1}$ & $6 \times 1 / 5$ \\
\hline$D_{\text {in }}$ & Half saturation intake rate of vole & ppm & 100 \\
\hline$H_{V}, H_{M}, H_{F}$ & Hill's coefficient dose-response curve & n.d. & 4 \\
\hline$L D_{50, X}$ & Lethal Dose for $50 \%$ of individuals of $\mathrm{X}$ & ppm & see Text \\
\hline$\eta_{M}$ & AR uptake rate in mustelids & n.d. & 0.5 \\
\hline$\eta_{F}$ & AR uptake rate in foxes & n.d. & 0.5 \\
\hline$k_{\text {out }, V}$ & Excretion rate of AR by voles & day $^{-1}$ & 0.4 \\
\hline$k_{\text {out }, M}$ & Excretion rate of AR by mutelids & day $^{-1}$ & 0.6 \\
\hline$k_{\text {out }, F}$ & Excretion rate of $\mathrm{AR}$ by foxes & day $^{-1}$ & 0.6 \\
\hline
\end{tabular}




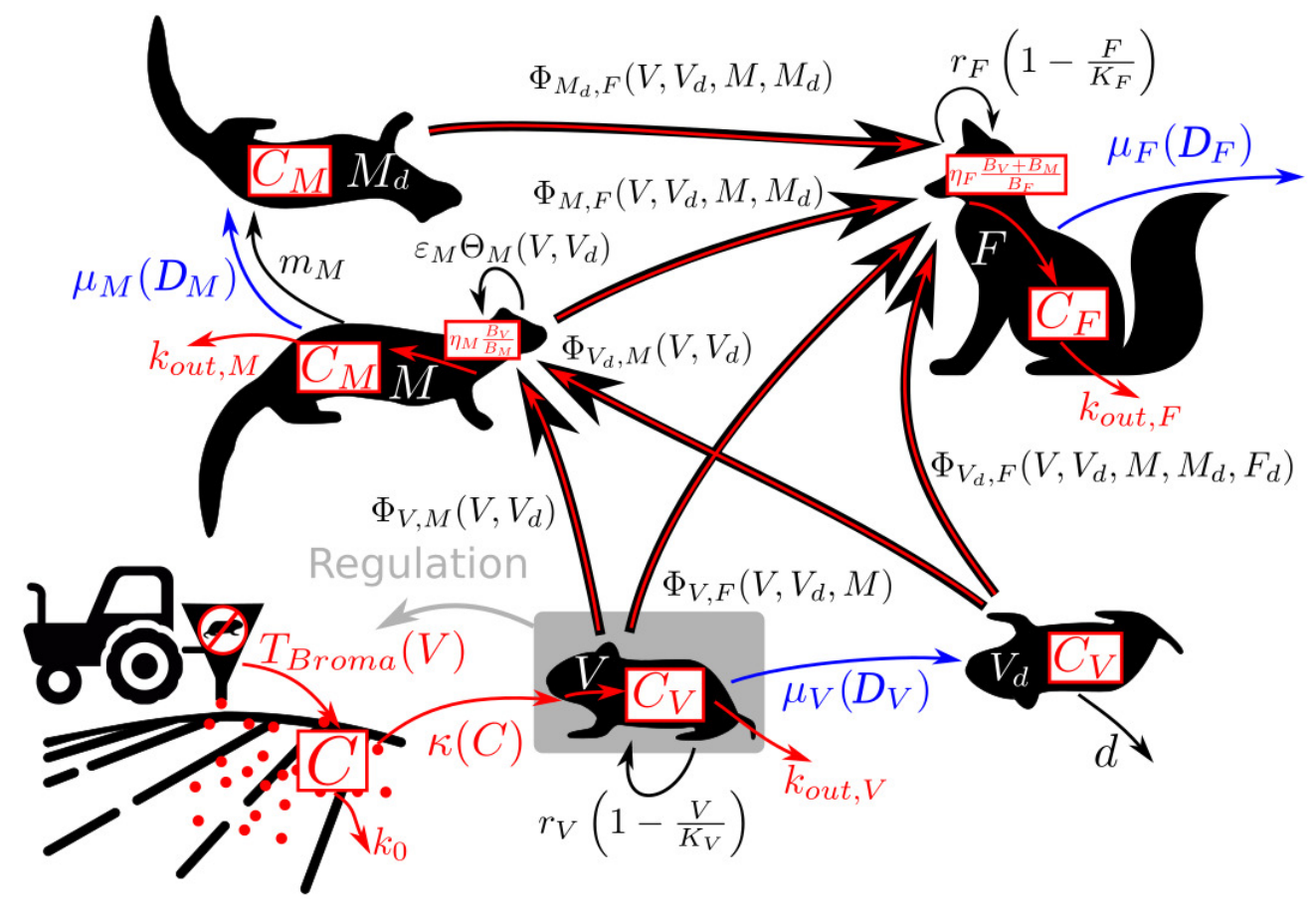

Figure 1 - Flow diagram of the fate and impacts of anticoagulant rodenticide (AR, bromadiolone) in a tri-trophic food web. Black arrows and equations correspond to natural dynamics with trophic interactions, red arrows and equations represent the transfer of AR through the system and its accumulation into the different compartments. The arrows and equations in blue correspond to its impact (i.e., death of individuals) on the three species populations. 


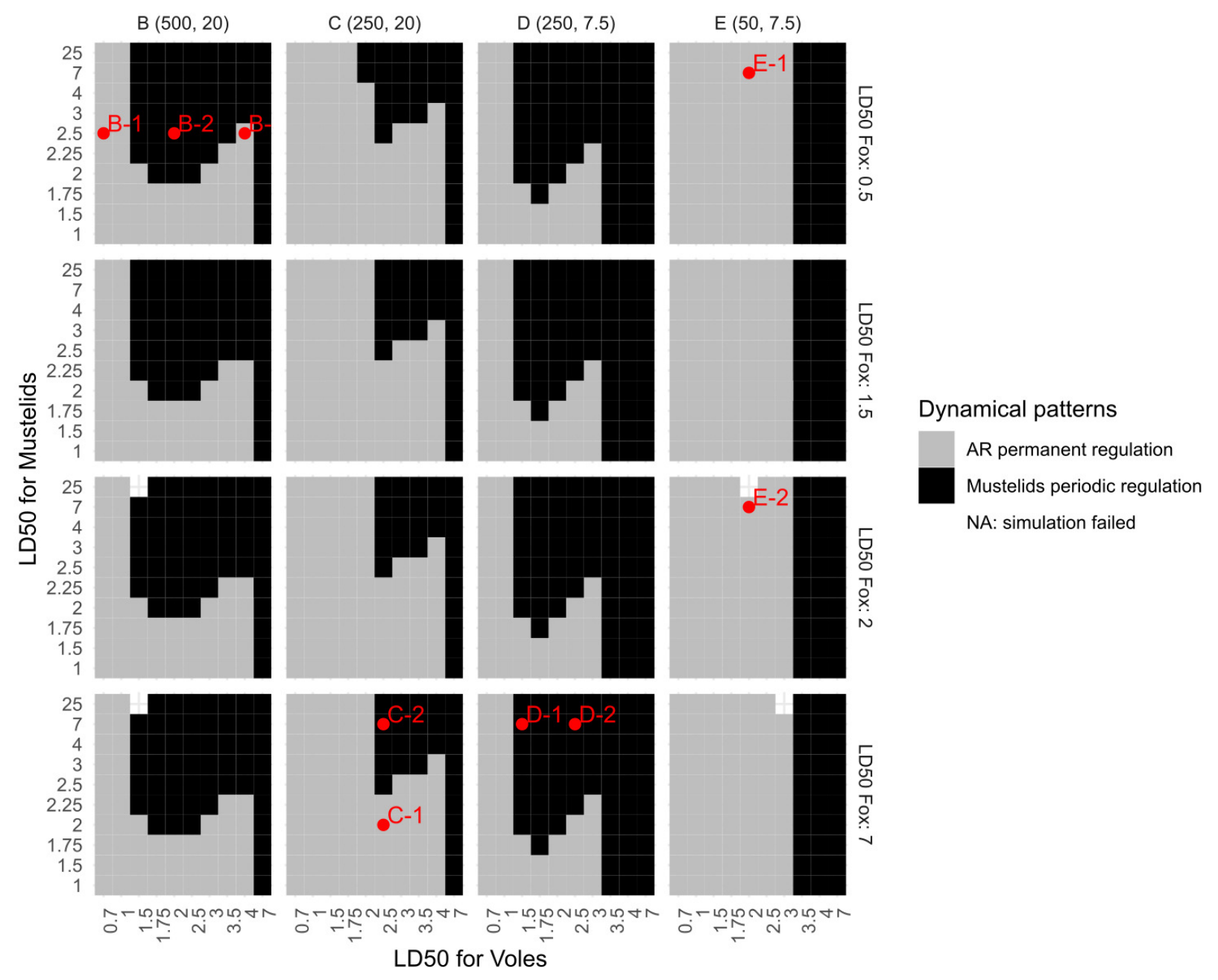

Figure 2 - Global sensitivity analysis of Lethal Dose for voles, mustelids and predators on the population dynamics of mustelids: "Mustelids periodic regulation" (see Figure 3,c,f,g,h) or "Permanent AR regulation" (see Figure 3-b,d,e,i,j). Points are simulations in Figure 3 . 


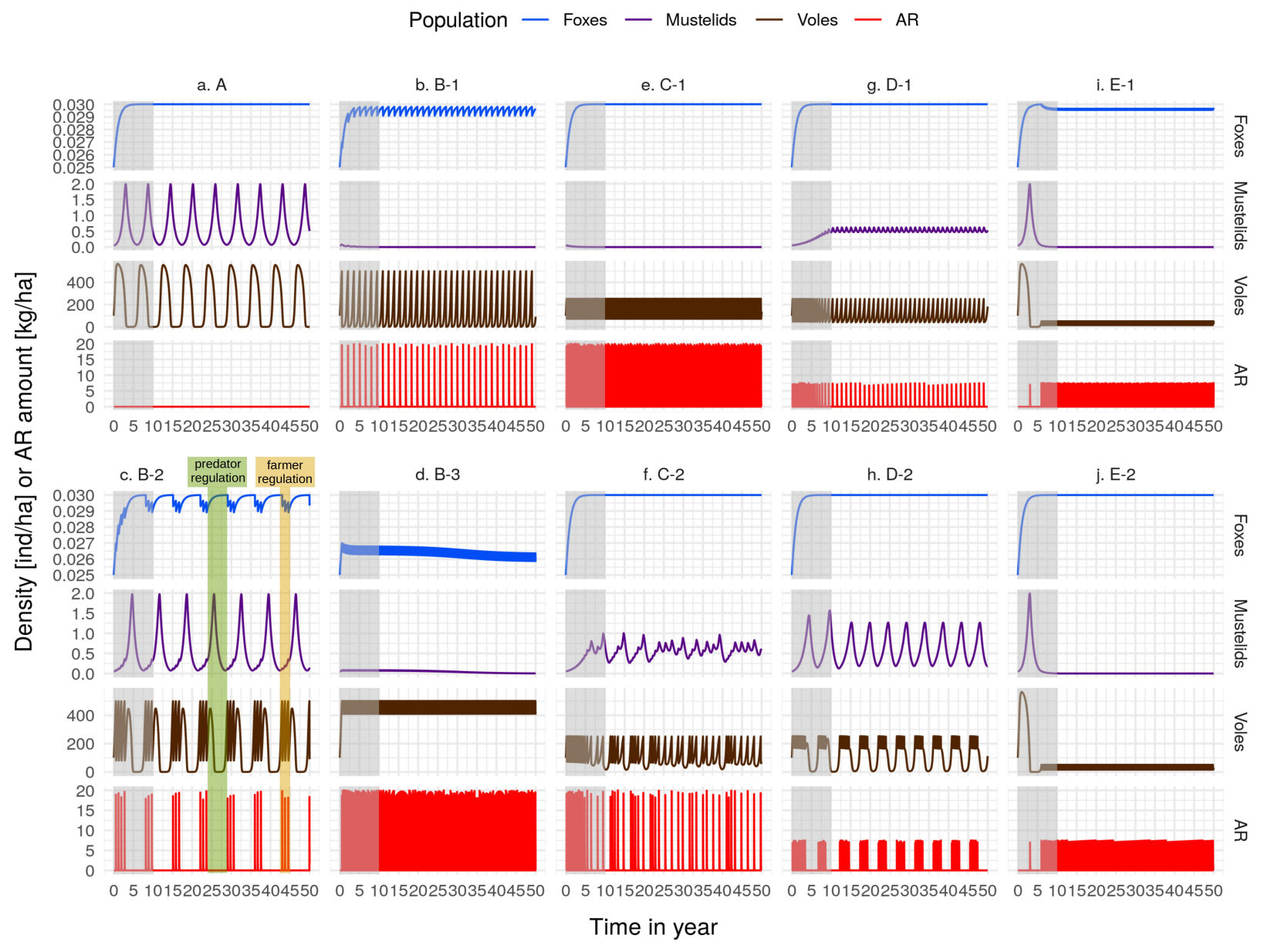

Figure 3 - Variation of population densities simulated over 50 years (10 years of burn-in period in grey area). Capital letter corresponds to farmer functional response: A the null model (no AR), B (vole threshold: 500in/ha, AR 20kg/ha), C(250,20), D(250, 7.5) and E(50,7.5). Numbers correspond to points in Figure 2, Panel c (B-2) illustrates predator-regulation and farmer-regulation phases, that can also be found in other graphics (C-2, D-1, D-2). 

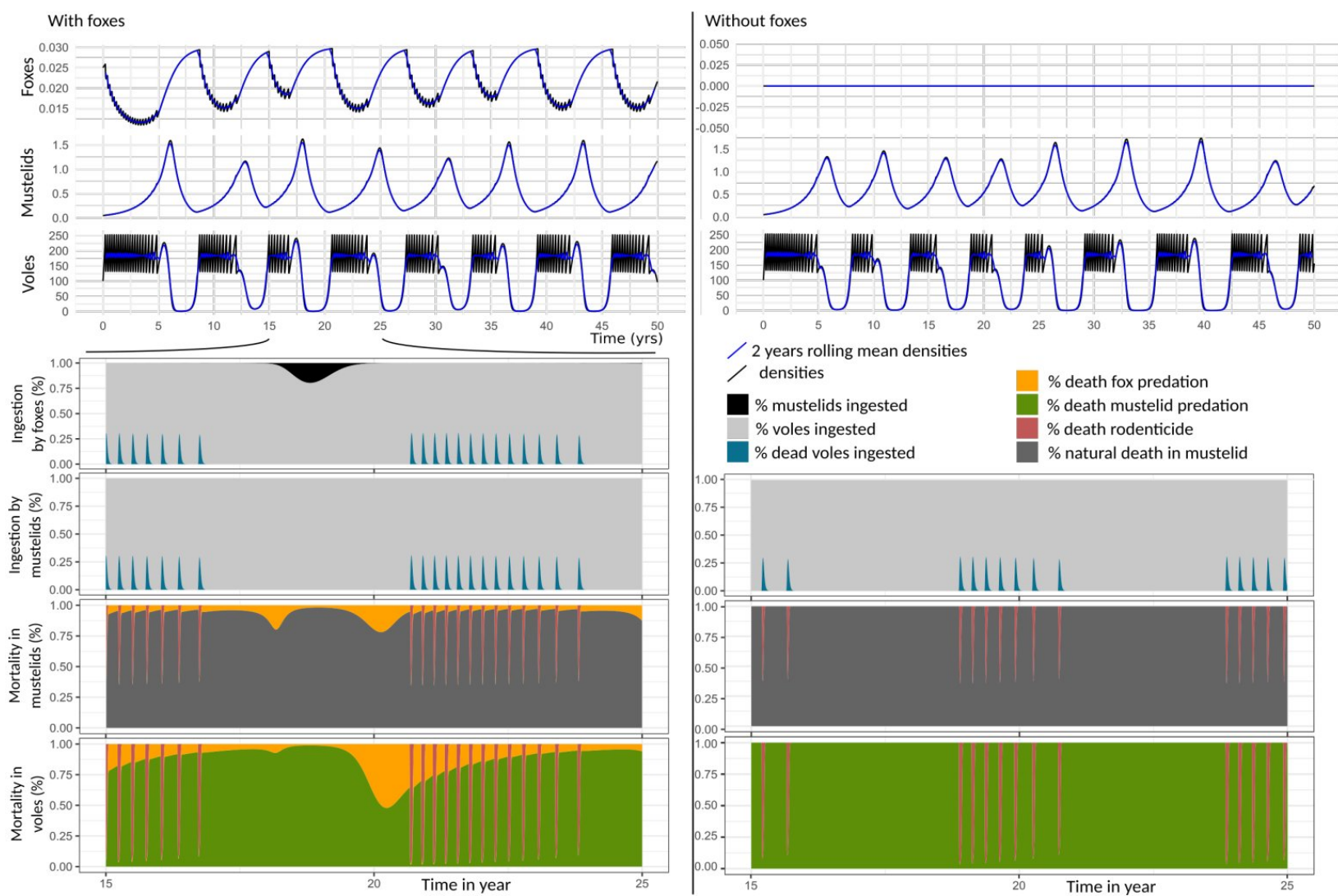

Figure 4 - Effects of intraguild predation. Top panels: daily densities of voles (in black) and 2-years rolling means of densities (in blue) for the farmer functional response d (i.e., $7.5 \mathrm{~kg}$ of rodenticide at threshold density of 250 voles) with and without foxes (left and right respectively). Bottom graphics: stacked charts of ingestion and mortality proportion. 


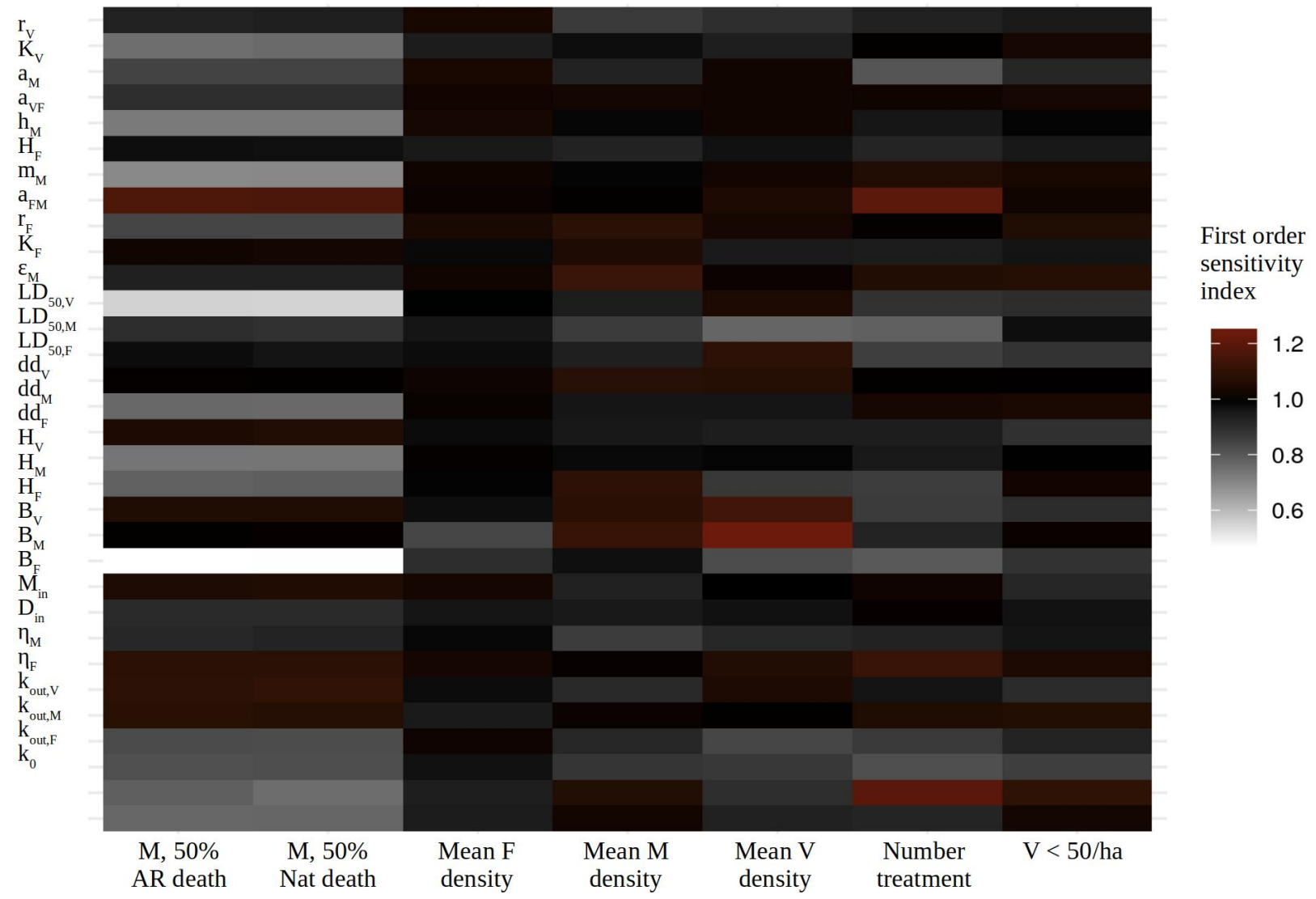

Figure 5 - Local sensitivity analysis: First-order Sobol's sensitivity index (denoted $S_{p}$ for parameter $p$ ) providing the global sensitivity of cost functions to parameters of the model (Sobol and Saltelli, 1993) for the model d (i.e., $7.5 \mathrm{~kg}$ of AR at threshold density of 250 voles). Analysis is based on 4200 simulations. Values reflect the expected fractional reduction in the variance for each cost functions that would be achieved if a specific parameter is fixed. Values of $S_{p}$ may be greater than one due to the potential correlation between variables. 